\title{
ARTIGOS
}

\section{CROWD-DESIGN COMO ALTERNATIVA DE PRODUÇÃO DISTRIBUÍDA: UM ESTUDO DE CASO EX-POST-FACTO EM EMPRESA DE MÓVEIS EM PALLET}

\author{
Alexandre Oliveira (UFPR); Isadora B. Dickie (UFPR); \\ Aguinaldo dos Santos, Ph.D (UFPR)
}

PALAVRAS CHAVE

Crowd-Design; inovação aberta; produção distribuída.

KEY WORDS

Crowd-Design; open innovation; distributed production.

\section{RESUMO}

Este artigo apresenta um estudo de caso ex-post-facto do projeto Sustainability Maker Brazil, onde foi possível analisar criticamente a participação de uma empresa do setor moveleiro com relação às práticas e às diretrizes da produção distribuída. Para tanto, além de uma Revisão Bibliográfica Sistemática através da qual foi possível conhecer e estabelecer estas práticas e diretrizes, também foram analisados os relatórios do projeto SuM/BR e suas referentes etapas no desenvolvimento de um novo produto. Como resultado, é possível observar que o Crowd-Design pode ser considerado como uma opção para a produção distribuída.

\section{ABSTRACT}

This paper presents an ex-post-facto study case made at Sustainability Maker Brazil's project. It was possible to critically analyze the participation of a furniture company with regard to practices and guidelines of distributed production. To this end, in addition to a Systematic Literature Review through which it was possible to meet and establish these practices and guidelines, were also analyzed the SuM/BR project reports and know the product development phases. As a result, it is possible to observe that the Crowd-design can be considered as an option for distributed production. 


\section{INTRODUÇÃO}

O presente artigo analisa o caso de desenvolvimento de novos produtos através do processo aberto de Crowd-Design, no âmbito do projeto Sustainability Maker Brazil (SuM/BR), através de um estudo de caso ex-post-facto. $O$ projeto Sustainability Maker é uma iniciativa internacional que, a partir de uma plataforma online baseada nos princípios da inovação aberta, busca facilitar a conexão de pessoas que podem contribuir de maneira significativa na resolução de problemas relacionados à sustentabilidade. Este projeto é liderado pela E-Concept (Alemanha) e o desenvolvimento da plataforma é financiada pela Comunidade Européia através do programa LIFE (LIFE11 ENV/ DE/000342). No Brasil este projeto está sendo desenvolvido pelo Núcleo de Design e Sustentabilidade da Universidade Federal do Paraná (NDS/UFPR).

O SuM/BR - o caso brasileiro do projeto - conta com a participação de duas empresas parceiras cuja função é apoiar todas as etapas de desenvolvimento e de produção da solução - neste caso, a Soliforte e a EcoDesign. A primeira atua no desenvolvimento de produtos oriundos da reciclagem de materiais da construção civil e a segunda desenha, produz e comercializa móveis em madeira fabricados a partir de pallets reutilizados. O presente artigo avalia de forma crítica a participação desta segunda empresa - a Eco-Design —-tendo como base as práticas e as diretrizes levantadas por uma revisão bibliográfica sistemática (RBS) acerca da produção distribuída por meio do Crowd-Design.

Nesse sentido, Crowd-design é uma modalidade de crowdsourcing, para o desenvolvimento de novos produtos e ideias na área do Design. O Crowd-Design utiliza os conhecimentos e recursos disponíveis na multidão para a resolução de problemas ou criação de conteúdo, geralmente pela internet, sendo esta participação podendo ser remunerada ou não (DICKIE et. al, 2014).

Como dito, este modelo de processo se origina do crowdsourcing, que de acordo com Estellés-Arolas e González-Ladrón-de-Guevara (2012), é um tipo de atividade participativa e online no qual um indivíduo, instituição ou organização propõe uma tarefa a um grupo heterogêneo e indefinido, via chamada aberta. O cumprimento dessas tarefas sempre traz um benefício para ambas as partes, podendo este benefício ser de variadas formas.

O crowdsourcing possui variações que dependem da atividade desenvolvida. O Crowd-Design é um deles, e que por sua vez, pode utilizar-se de outras duas variações, a saber:
- Crowdfunding: que corresponde ao financiamento de projetos por meio da multidão. Consiste em uma solicitação pública para o financiamento específico de um projeto. Com isso, pequenas contribuições feitas por indivíduos isolados são adicionados ao todo para a implementação do projeto (BANNERMAN, 2013);

- Crowdvoting: que corresponde ao processo de seleção de alternativas feitas pela multidão que geralmente ocorre de forma online buscando a opinião de um grande número de pessoas sobre um tópico específico (BEHREND et al., 2011).

Assim como as atividades do crowdsourcing, a produção distribuída aumentou a capacidade dos usuários interferirem no que é produzido, desde a personalização de produtos até a fabricação pessoal (KOHTALA, 2015). O termo "produção distribuída", na engenharia, traz a perspectiva de um planejamento de produção entre uma rede de empresas visando agilidade, flexibilidade, fabricação voltada ao consumidor, entre outros (Bruccoleri et al., 2005; Leitão, 2009; Tuma, 1998).

De acordo com Kohtala (2015), "produção distribuída" é um termo usado mais ideologicamente do que epistemológicamente quando se trata de modelos alternativos de negócios e oportunidades socialmente benéficas para produção e um consumo mais responsivo. No Design, Kohtala (2015) defende que este conceito está diretamente ligado à participação do usuário no desenvolvimento do projeto ou no fato deste ser orientado para o consumidor final.

O conceito de produção distribuída ainda é difuso e muitas vezes é definido por ações pontuais como o ato do consumidor fazer parte da produção, conhecido como prosumption (Benkler, 2006). Kohtala (2015) traça uma revisão bibliográfica da produção distribuída a partir da sustentabilidade e Lin et al. (2012) analisa a "produção colaborativa" em pequenas e médias empresas. Outros termos como "personal manufacturing" (Bauwens et al., 2012) ou "making" (Gauntlett, 2013) também são citados no contexto de alusão à produção distribuída.

\section{PROCEDIMENTOS METODOLÓGICOS E DESENVOLVIMENTO}

\subsection{Procedimentos da pesquisa}

Como mencionado anteriormente, este estudo refere-se a um estudo de caso ex-post-facto, cujos dados coletados são, majoritariamente, de cunho qualitativo. Para tanto, foram utilizados os seguintes procedimentos: (1) Re- 
visão Bibliográfica Sistemática (RBS), que auxiliou no levantamento de informações para maior entendimento e elucidação das etapas no que tange a produção distribuída e o Crowd-Design; (2) Coleta de documentos, como relatórios do projeto SuM/BR, fornecidos pela equipe do NDS/UFPR que participou do SuM/BR; (3) Análise comparativa das ferramentas e métodos utilizados no projeto SuM/BR a partir dos dados fornecidos pela RBS afim de avaliar o processo.

Utilizando o método da RBS proposto por Conforto et al. (2011), objetivou-se conhecer estudos sobre as práticas de desenvolvimento de novos produtos por meio do Crowd-Design e crowdsourcing em organizações, assim como a relação das etapas dentro do processo de desenvolvimento de produtos (PDP). Também foi usada a RBS para levantar informações sobre produção distribuída e suas práticas no processo de desenvolvimento de novos produtos (PDP).

De acordo com Conforto et al. (2011), a primeira etapa de uma RBS é a definição do problema no qual se busca responder uma ou mais perguntas. Após o estudo de caso feito com o projeto SUM/BR e a EcoDesign, houve a necessidade de se obter informações de cunho comparativo para analisar e entender quais práticas foram as mais eficientes e quais os pontos positivos e negativos no projeto. Logo, a principal questão seria avaliar de forma crítica as práticas no estudo de caso deste modelo de Crowd-Design para o desenvolvimento de novos produtos. Como objetivos específicos: procurou-se a definição de uma estrutura no PDP das organizações usando a multidão como fonte criativa, para servir de base comparativa ao caso estudado; e de que forma o Crowd-Design pode ser uma alternativa sustentável de produção distribuída para as organizações.

Após esclarecidos os objetivos e tendo como fonte primária os relatórios do projeto SUM/BR (2014) e suas referências subsequentes, houve a definição dos strings de busca, assim como proposto por Conforto et al. (2011). Os strings de busca foram divididos em duas categorias. A primeira foi para se entender o processo de desenvolvimento de produtos por meio do Crowd-Design nas organizações e a segunda categoria de strings buscou-se a abordagem da produção distribuída no PDP das organizações. A relação dos strings usados, assim como a classificação e o número de artigos encontrados está no Apêndice A.

Os critérios de inclusão e qualificação, de acordo com Conforto et al. (2011), são importantes para verificar similaridade e rigorosidade nas referências buscadas. Para isso, como critérios de inclusão foi definido que seria usada a plataforma ScienceDirect (2015). Os seguintes critérios foram considerados: "em qualquer local da publicação" que "contém" os strings de busca; no recorte de tempo foram considerados os trabalhos "publicados nos últimos 10 anos"; "somente artigos"; "somente no idioma inglês". Os critérios de qualificação, que atestam a importância do artigo, foram levados em consideração pelo interesse em publicações que descrevessem os métodos de desenvolvimento de produtos usando o Crowd-Design e a multidão, estudos de caso na área e exemplos práticos.

A seleção dos artigos para a RBS foi feita da seguinte forma: leitura do título, resumo e leitura completa dos artigos, verificando entre cada etapa a congruência dos dados em razão aos critérios supracitados assim como propõe Conforto et al. (2011).

\subsection{Crowd-Design como opção para a produção distribuída}

Dos 24 artigos encontrados e analisados na RBS, nenhum menciona o termo "Crowd-Design" como modalidade de desenvolvimento de novos produtos via crowdsourcing. Apenas a fonte primária define como Crowd-Design o PDP com a multidão. Entretanto, Djelassi e Decoopman (2013) citam que a prática do crowdsourcing no desenvolvimento de produtos nas empresas primeiramente consiste em integrar o consumidor (ou desenvolvedor externo) no processo de inovação, ou seja, trazer a figura do consumidor para fazer parte do processo de produção. Toffler (1980) denomina este sujeito participante de "prosumer".

Kohtala (2015) em sua revisão bibliográfica acerca da produção distribuída e sustentabilidade cita que a maioria dos autores voltados para o design de produto abordam o assunto pelo prisma da personalização de produtos via produção digital, com diversas aproximações. Dentre elas, o design aberto (open source) para os próprios consumidores modificarem seus produtos e o fornecimento de medidas corpóreas para a customização focada no consumidor. Nesta RBS, a maioria dos artigos (07 de 11) também trata destes temas, e principalmente em como a produção voltada às necessidades do consumidor podem gerar produtos menos efêmeros consumidos pelos usuários e consequentemente com maior proximidade à sustentabilidade (Diegel et al. 2010).

Partindo desta proposição, alguns autores como Kohtala (2015) citam a co-criação como fonte de produ- 
ção distribuída, porém, em nenhum deles cita o crowdsourcing ou o Crowd-Design como alternativa.

Djelassi e Decoopman (2013) citam que o fato da empresa confiar em seus clientes no PDP traz para estes uma sensação de importância e auto-estima, se orgulhando de fazer parte no processo e de saber que a empresa está contando com suas soluções. Os mesmos autores defendem que um produto desenvolvido por meio da multidão resulta em produtos mais adaptados à necessidade do consumidor e consequentemente produtos mais longevos.

No crowdsourcing, Brabham (2010) defende que a boa prática desta modalidade começa na elaboração de uma tarefa (ou desafio) bem planejada. O mesmo autor define nove princípios norteadores para a elaboração de uma tarefa e a boa execução de um projeto que envolve a multidão como fonte criativa ou solucionadora:

1) definição clara do problema e comunicar as soluções que são esperadas;

2) determinar o nível de comprometimento da empresa com os resultados obtidos, no sentido de explicitar o tipo de solução que será produzido e porquê;

3)entender a motivação para a participação dos usuários;

4) investir em um site ou plataforma que é utilizável, interessante e bem concebido;

5) ter um plano promocional e um plano para o crescimento da comunidade;

6) ser honesto, transparente e ágil;

7) não controlar o comportamento da multidão, pois ela não está sendo consultada para realizar os objetivos organizacionais e sim convidada a se envolver no processo de desenvolvimento de produtos;

8) reconhecer os usuários com as premiações que Ihe foram prometidas; $\mathrm{e}$

9) avaliar o projeto sob vários ângulos, solicitando sempre o feedback dos participantes.

Ainda sobre a elaboração da tarefa, Djelassi e Decoopman (2013) ressalta que para qualquer operação dentro do crowdsourcing, a organização deve explicar claramente as regras e o que se busca com tal prática para não haver nenhum desentendimento e consequentemente a sensação de trapaça ou exploração por parte dela. Esta sensação deve ser evitada em todo o processo de desenvolvimento.

O processo de crowdsourcing, no ponto de vista das organizações, se for bem conduzido pode trazer diversos benefícios às empresas. Alonso (2013) salienta que esta prática oferece uma flexibilidade em projetos e que deveria fazer parte do processo de desenvolvimento. Zhao e Zhu (2011) enfatiza que a organização participante precisa definir claramente o que ela está buscando com este tipo de modelo e Malone et al. (2010) propõe a visualização prévia de como esta organização vai obter os resultados esperados. Tais ações podem influenciar no tipo de interação que a mesma terá com a multidão e que tipo de aproximação ela terá.

\subsection{As etapas do processo de Crowd- Design aplicadas ao projeto SuM/BR e a participação da empresa parceira}

A análise dos relatórios do projeto SuM/BR foi realizada pelos três autores deste artigo, sendo o conteúdo analisado, principalmente, as etapas do processo de Crowd-Design e a relação e a participação da empresa parceira, a EcoDesign. Na figura 01 é possível visualizar as etapas do processo de Crowd-Design tal como aconteceram no caso estudado. Estas etapas são sugeridas pelo projeto Sustainability Maker.

De acordo com o apresentado na figura 01, a etapa inicial do processo de Crowd-Design corresponde ao levantamento do problema. Para isso, a equipe do projeto SuM/BR foi a campo coletar dados sobre a Comunidade Águas Claras, em Piraquara/PR. Esta comunidade serviu de fonte de dados para a descoberta do desafio - ou seja, qual seria o problema que seria levado para a multidão solucionar, através da plataforma Innonatives (2015). Neste tipo de abordagem do problema, prioriza-se o modelo botom-up, onde procura-se entender as reais necessidade dos usuários, adaptando-a a estratégia de negócios da empresa. Assim, nesta etapa a empresa EcoDesign foi consultada e mostrou-se interessada em direcionar seus produtos também para o público de baixa-renda. A etapa seguinte foi a de votação. A equipe do projeto SuM/BR após analisar os dados coletados, voltou à comunidade de Águas Claras e apresentou quatro principais problemas evidenciados pela coleta de dados. A comunidade, então, pode votar naquele que mais the era relevante; no caso, melhorar a socialização nas cozinhas de suas casas. É importante ressaltar que, sem o auxílio da equipe do projeto SuM/BR, a empresa EcoDesign talvez não tivesse oportunidade para investir (tempo e dinheiro) num estudo aprofundado, tal qual o realizado junto à comunidade de baixa-renda. A figura 02 apresenta as etapas da coleta de dados e da votação desta primeira etapa do processo de Crowd-Design. 
Figura 01 -Etapas do processo de Crowd-Design do projeto SuM/BR

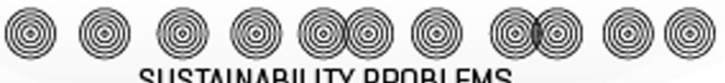

SUSTAINABILITY PROBLEMS

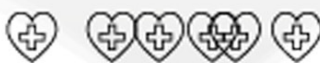

CROWD VOTING

CHALLENGE

品 品㫛品

CROWD SOURCING

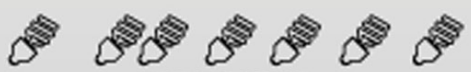

SOLUTIONS

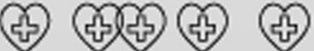

CROWD VOTING

28⿱⺈

EXPERT PANEL

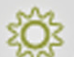

BEST SOLUTION

Assim, com o problema escolhido pela comunidade, o próximo passo foi o de definir o desafio, de maneira a gerar um briefing para disponibilizar na plataforma Innonatives. A figura 03 mostra o briefing disponibilizado na plataforma para os participantes.

Para a realização do briefing, a empresa EcoDesign foi novamente envolvida, sendo de extrema importância as informações fornecidas pela mesma com relação ao seu processo de fabricação. Pois, tendo em vista que o desafio consistiu no desenvolvimento de um produto para auxiliar na socialização em cozinhas de famílias de baixa-renda, o mesmo deveria ser desenvolvido de maneira a ser produzido pela empresa parceira.

As etapas seguintes consistiram do envio da ideia, conceito e solução ao desafio proposto. Ao final de cada uma das etapas, foi realizada a votaçao online - crowdvoting - e também a postagem de comentários em cada ideia, conceito e solução enviada. Em todas estas etapas, a empresa EcoDesign também foi envolvida, realizando votos e comentando, como pode ser visto na figura 04.

Do crowdvoting da solução, ou seja, a última etapa de votação para a escolha do vencedor, sairam quatro vencedores: um terceiro lugar, dois segundo lugares e um primeiro lugar. $O$ produto que ganhou em primeiro lugar foi o produzido pela empresa EcoDesign. A figura 05 mostra o projeto do produto e o mesmo após ser produzido.

No caso do projeto SuM/BR, por se tratar de um piloto, e por ter desde o início o suporte de duas empresas 
Figura 02 -Etapas da Fase de Coleta de Dados do processo de Crowd-Design do projeto SuM/BR

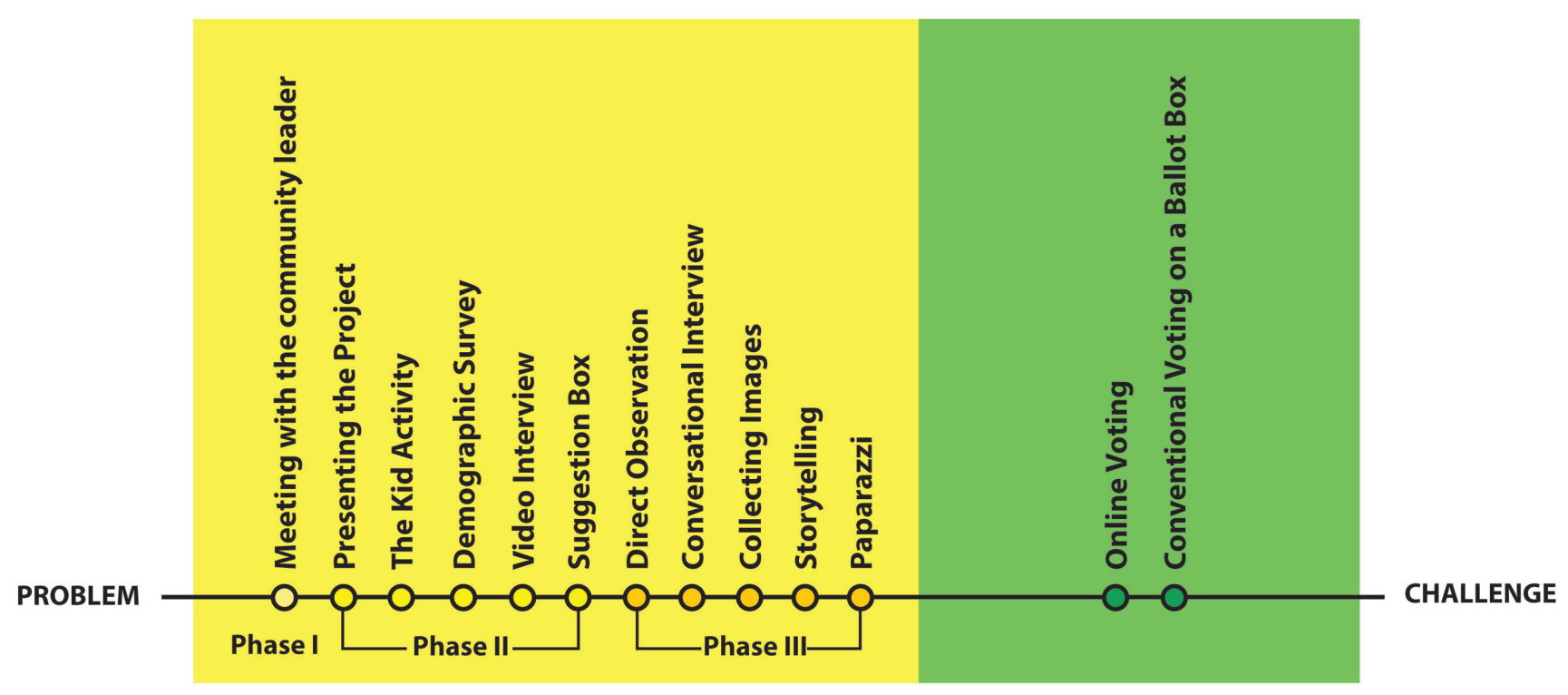

Fonte: Relatório do projeto SuM/BR (2015, web).

Figura 03 -Briefing do projeto SuM/BR.

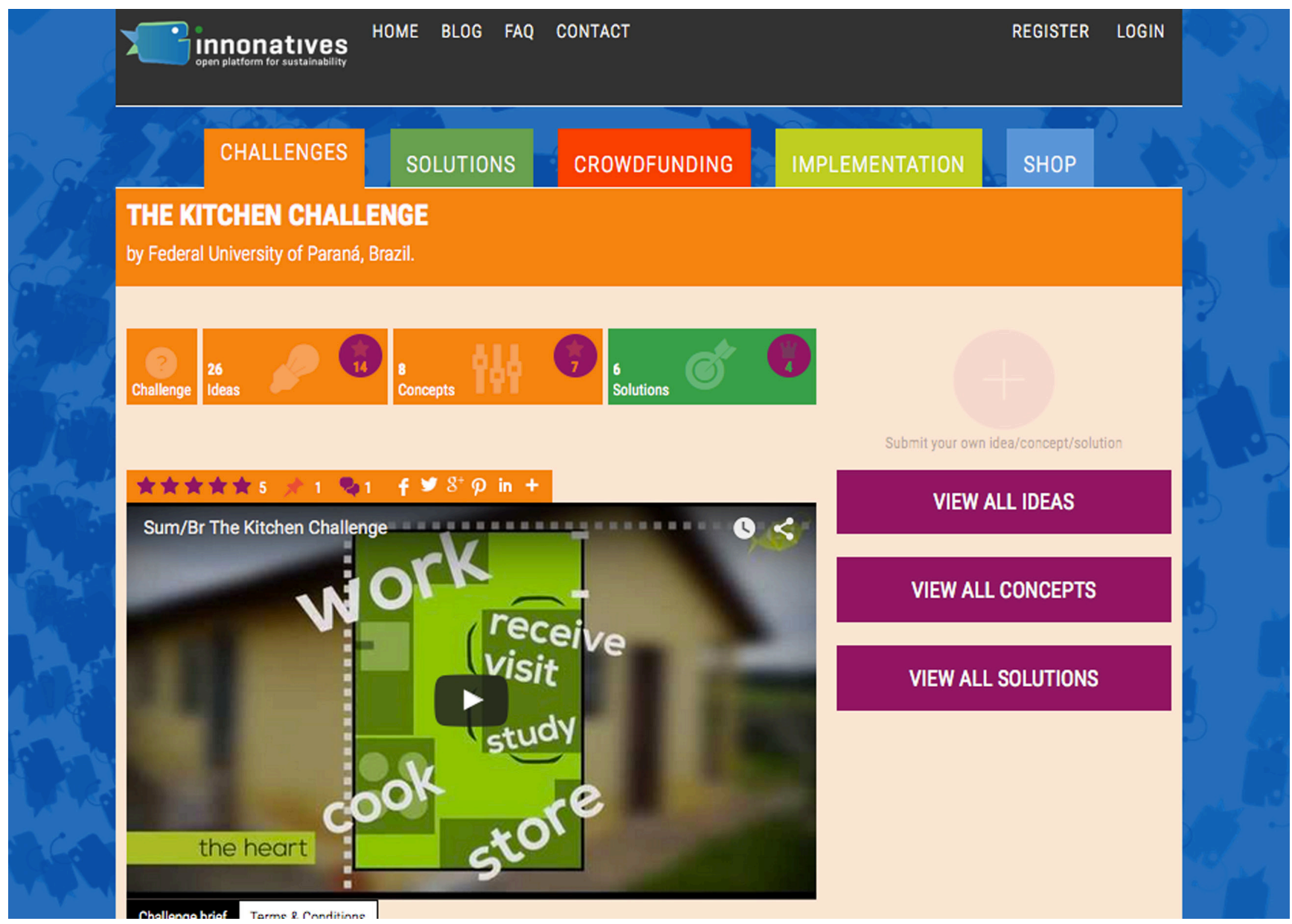

Fonte: Innonatives platform (2015, web). 
Figura 04 -Comentário em uma das ideias enviadas para o desafio do projeto SuM/BR pela empresa parceira EcoDesign.

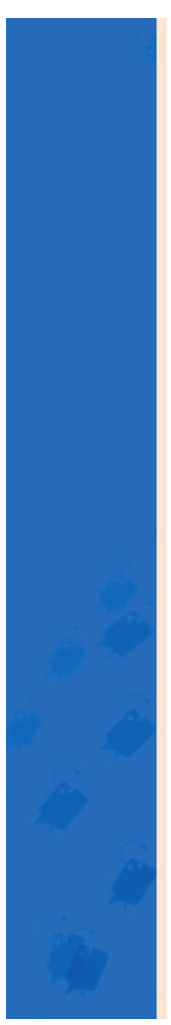

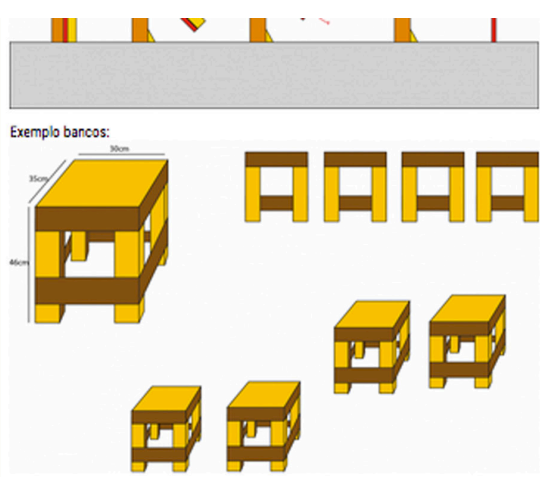

Ps: As cores da peça 1, o arreíato principal, sabo ilustrativas, elas quando fabricades văo seguir o padrão de cor

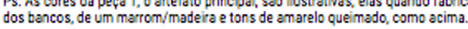

COMMENTS

B.

Dinho 28-11-2014 13:41

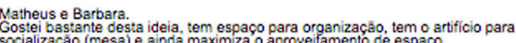

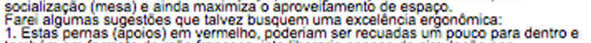

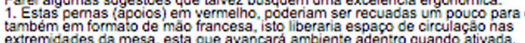

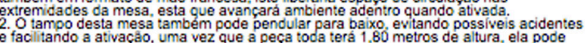

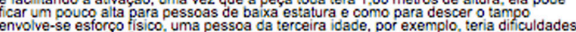

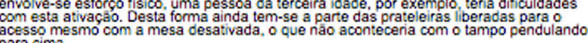
para Comat o tampo pendulando para baixo, para nâo perder a ideia do banco, quando a

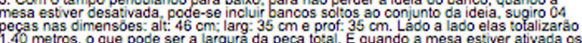

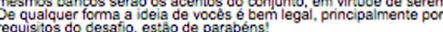

Matheus Tureck 28-11-2014 $15: 26$

Muito obrigado pelas dicas! As mudancas seráo avaliadas e feitas sim, nâo

havia pensado na possibilidadeda da mesa sendular para baixo, com isso,
realmente ela se adequa melhor a ideia do projeto. Obriacol

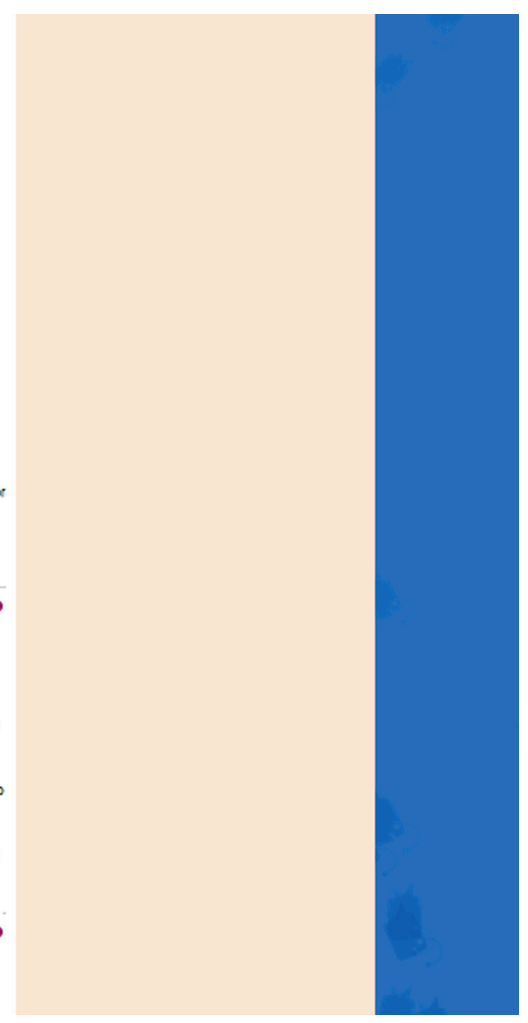

Fonte: Innonatives platform (2015, web)

Figura 05 -Rendering do móvel (esquerda); móvel produzido pela empresa EcoDesign (direita).
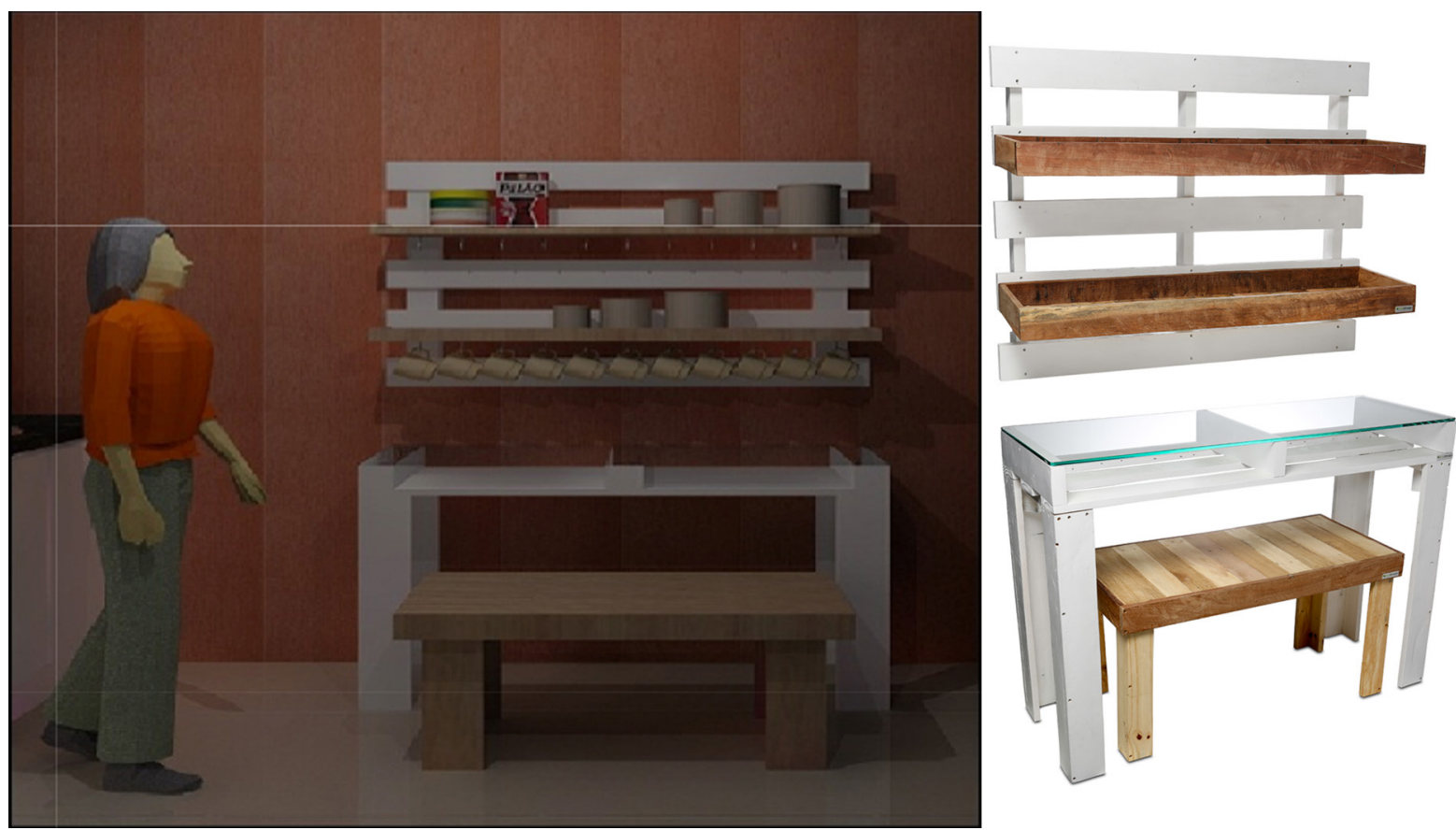

Fonte: innonatives platform (2015, web). 
parceiras - sendo uma delas a EcoDesign que produziu a solução vencedora do desafio — não foi necessário utilizar a etapa de crowdfunding, bem como o marketplace ou auction, como previsto no processo original do projeto Sustainability Maker e na plataforma innonatives.

Com relação a implementação da solução, a empresa EcoDesign fez a doação de um exemplar para ser sorteado na comunidade de Águas Claras como forma de agradecimento pela participação no projeto. A figura 06 mostra como o móvel já está sendo utilizado pela família contemplada no sorteio.
A multidão como fonte de ideias e soluções é a premissa para que o Crowd-Design funcione de maneira a se obter melhores resultados para os consumidores (que participam ou não do processo) e para as organizações. Resultados que podem variar de um produto mais adaptado às necessidades do consumidor para um produto mais econômico no ponto de vista do PDP das organizações, entre diversas outras situações. Tal afirmação pôde ser analisada no estudo de caso estudado: o processo de Crowd-Design no projeto SuM/BR gerou um produto que foi amplamente usado pelo público-alvo, público este que

Figura 06 -Utilização do móvel pela família de baixa-renda contemplada pelo sorteio.
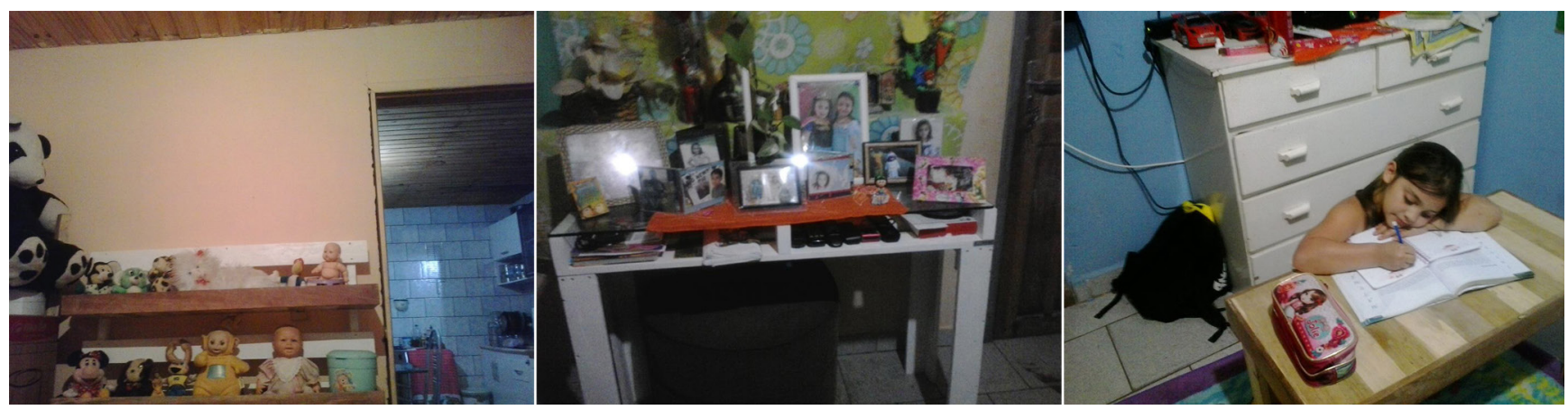

Fonte: Arquivo pessoal.

Interessante perceber que, mesmo tendo sido planejado para a cozinha, o móvel, quando implementado, recebeu outro fim; na verdade, está sendo utilizado para três novas funções: a parte das prateleiras está sendo utilizada para guardar brinquedos; a parte da mesa (ou do balcão) está sendo utilizada como aparador para suporte a objetos pessoais, como porta retratos; e o banco está servindo de mesa para a criança estudar.

\section{CONCLUSÃO}

Este artigo apresentou um levantamento de diretrizes para as práticas e competências do crowdsourcing em projetos crowd-design nas organizações. O levantamento das informações trouxe características que definem o que é uma boa conduta para as empresas quando se trata desta nova modalidade de desenvolvimento de produtos e soluções. Para isso, o método usado foi a Revisão Bibliográfica Sistemática, que se mostrou eficaz tanto na organização quanto na seleção das pesquisas para este trabalho. A sistematização das etapas facilita o manuseio de dados na quantidade que foi gerada e analisada, evitando o retrabalho e agilizando a pesquisa. participou do desenvolvimento.

De acordo com as etapas levantadas na RBS para uma boa execução no processo de crowdsourcing, cabe salientar que no projeto SuM/BR as fases de definição do desafio e a parte informacional do projeto (diretrizes 1 e 2) foram bem executadas pela equipe. O processo descrito de coleta de necessidades com diversas entrevistas e análises intensifica o papel da organização em tentar entender o que o consumidor precisa e o que a empresa define como essencial na produção. Mesmo este consumidor sendo um prosumer, é papel das organizações entender quais necessidades e problemas são relevantes para a sua produção e consequentemente oferta. Por isso, é importante e necessário o papel de ambas as partes no processo de desenvolvimento de produto voltado às necessidades do consumidor: uma para definir o que se precisa (usuário) e outro para definir parâmetros de produção e consumo (organizações).

Nas etapas de desenvolvimento da ideia e avaliação, o papel da empresa EcoDesign deu-se não apenas pela aceitação ou rejeição de soluções, mas sim com avaliações de modo a adaptar as ideias ao processo produtivo 
da organização ou demonstrando maleabilidade e abertura às soluções excêntricas ou dissonantes ao habitual no setor moveleiro. Em nenhum momento foi explicitado a posição estratégica ou a visão da empresa em relação ao projeto. No briefing, as informações acerca da empresa eram somente de origem produtiva. Informações como o posicionamento de mercado desta empresa ou o que ela almeja com um tipo de processo deste cunho não foi passado para os participantes do desafio. Por mais que a interação e a construção coletiva tenha ocorrido por meio da plataforma Innonatives, esta conduta da empresa foi de caráter passivo, ou seja, em resposta às informações que vieram até a mesma.

Dentre todos os artigos sobre produção distribuída, nenhum cita o crowdsourcing como alternativa, entretanto, se os consumidores forem tratados como "multidão", o que de fato são, é possível transportar o conceito de Crowd-Design para o PDP de novos produtos sendo uma alternativa de produção distribuída.

Ainda que o crowdsourcing seja uma nova modalidade de geração de novas ideias e soluções e que muitas empresas estejam usufruindo disto, diferenciações de princípios heurísticos ainda devem ser explanados. Necessita-se elaborar o assunto no sentido de qual tipo de crowdsourcing e qual atividade dentro desta prática pode ser mais vantajosa em casos específicos na indústria de acordo com sua área de atuação, porte, e tipo de desenvolvimento que se almeja. E assim a própria organização poder comunicar aos participantes quais são os seus anseios e suas estratégias, dando mais base informacional para o desenvolvimento de novos produtos.

Existem diversos estudos analisando o papel do desenvolvedor em crowdsourcing nas áreas da psicologia e tecnologia da informação, mas é carente a pesquisa neste tema por parte do design. Descobrir suas competências voltadas especialmente no desenvolvimento de novos produtos e a diferença do desenvolvimento convencional de novos produtos para esta forma seriam alguns dos caminhos a serem traçados futuramente para um maior entendimento no processo de crowdsourcing em projetos de crowd-design nas empresas.

\section{AGRADECIMENTOS}

Os autores agradecem o apoio financeiro da CAPES (Coordenação de Aperfeiçoamento de Pessoal de Nível Superior) pela bolsa de mestrado demanda social.

\section{REFERÊNCIAS}

1. BANNERMAN, S. Crowdfunding Culture. In: Journal of Mobile Media, v. 07, n. 01, p. 1-30, 2013.

2. Corporation, Santa Monica, CA.

3. BAUWENS, M., et al. A Synthetic Overview of the Collaborative Economy. Orange Labs and P2P Foundation. 2012.

4. BEHREND, et al. The viability of crowdsourcing for survey research. In: Behavior research methods, v. 43, n. 3, p. 800-813, 2011.

5. Collaborative Economy. Orange Labs and P2P Foundation.

6. BENKLER, Y., The Wealth of Networks: How Social Production Transforms Markets and Freedom. Yale University Press, New Haven, CA. 2006.

7. BRABHAM, D. C., Crowdsourcing As A Model For Problem Solving: Leveraging The Collective Intelligence Of Online Communities For Public Good . Department of Communication The University of Utah. 2010.

8. BRUCCOLERI, M., et al. Production planning in reconfigurable enterprises and reconfigurable production systems. CIRP Ann. e Manuf. Technol. 54, 433-436. 2005.

9. CONFORTO E.; et al., Roteiro para revisão bibliográfica sistemática: aplicação no desenvolvimento de produtos e gerenciamento de projetos . $8^{\circ}$ Congresso Brasileiro de Gestão de Desenvolvimento de Produto. Anais do $8^{\circ}$ CBGDP. 2011.

10. DICKIE, I. et al., O Processo de Desenvolvimento De Produtos Via Crowd-Design: oportunidades para a sustentabilidade . $11^{\circ}$ Congresso Brasileiro de Pesquisa e Desenvolvimento em Design, vol.1, num.4. p. 1268-1279. 2014.

11. DIEGEL, O., et al., Tools for sustainable product design: additive manufacturing. J. Sustain. Dev. 3, 68-75. 2010.

12. DJELASSI, S.; DECOOPMAN, I. "Customers' participation in product development through crowdsourcing: 
Issues and implications". Industrial Marketing Management, 42 (5), pp. 683-692. 2013.

13. ESTELLÉS-AROLAS, E.; GONZALEZ-LADRÓN-DE-GUEVARA, F., Towards an integrated crowdsourcing definition". Journal of Information Science, 38 (2) pp. 189200. 2012

14. teringof polyamidepowders:an energy perspective. J. Clean. Prod.18,1722-1730.

15. GAUNTLETT, D. Making Is Connecting: the Social Meaning of Creativity, from DIY and Knitting to YouTube and Web 2.0. John Wiley \& Sons, Hoboken, NJ. 2013.

16. INNONATIVES PLATFORM. Disponível em: https:// www.innonatives.com/challenge/sus-brazil/view. Acesso em agosto de 2015.

17. KOHTALA, C. "Addressing Sustainability in Research on Distributed Production: An integrated literature review." Journal of Cleaner Production. 10.1016/j.jclepro.2014.09.039. 2015.

18. LEITÃO, P. Agent-based distributed manufacturing control: a state-of-the-art survey. Eng. Appl. Artif. Intel. 22, 979-991. 2009.

19. LIN, H.W., et al.: Design of a Global Decision Support System for a manufacturing SME: Towards participating in Collaborative Manufacturing. Int. J. Production Economics 136, 1-12. 2012.

20.MALONE, T.; et al. 2010. "Harnessing crowds: Mapping the genome of collective intelligence." MIT Sloan School Working Paper (4732-09).

21. RELATÓRIO DO PROJETO SUSTAINABILITY MAKER BRAZIL. Disponivel em: http://www.designintothecrowd.com/sum-br/. Acesso em agosto de 2015.

22. SCIENCE DIRECT. Disponível em: http://sciencedirect. com/. Acesso em agosto de 2015.

23. SUSTAINABILITY MAKER PROJECT. Disponível em: http://sustainabilitymaker.org/. Acesso em agosto de 2015.

24. TOFFLER, A. The Third Wave. Morrow, New York. 1980.
25.TUMA, A. Configuration and coordination of virtual production networks. Int. J. Prod. Econ. 56-57, 641-648. 1998.

26. ZHAO, Y.; ZHU, Q. "Evaluation on crowdsourcing research: Current status and future direction". Information Systems Frontiers, 16 (3) pp. 417-434. 2012. 